\title{
Effect of Cisplatin on the Frequency and Immuno-inhibitory Function of Myeloid-derived Suppressor Cells in A375 Melanoma Model
}

\author{
Xiang Huang ${ }^{1}$, Dan Guan ${ }^{1}$, Yong-Qian Shu ${ }^{1}$, Lian-Ke Liu ${ }^{1}$, Fang Ni*
}

\begin{abstract}
Background: To investigate the change of frequency and immuno-inhibitory function of myeloid-derived suppressor cells (MDSCs) after treatment of cisplatin (DDP) in A375 human melanoma model. Materials and Methods: BALB/c nude mice were inoculated with $\mathbf{A 3 7 5}$ cells to establish the human melanoma model and randomly divided into control group given normal saline (NS) and experimental group treated with DDP (5 mg/ $\mathrm{kg}$ ). The percentages of MDSCs in the tumor tissue and peripheral blood after DDP treatment were detected by flow cytometry. The proliferation and interferon- $\gamma($ IFN- $\gamma)$ secretion of $T$ cells co-cultured with MDSCs were analyzed through carboxyfluorescein succinimidyl ester (CFSE) labeling assay and enzyme-linked immunospot (ELISPOT) assay, respectively. Results: In A375 human melanoma model, DDP treatment could significantly decrease the percentage of MDSCs in the tumor tissue, but exerted no effect on the level of MDSCs in peripheral blood. Moreover, DDP treatment could attenuate the immuno-inhibitory function of MDSCs. T cells co-cultured with DDP-treated MDSCs could dramatically elevate the proliferation and production of INF- $\gamma$. Conclusions: DDP can decrease the frequency and attenuate immuno-inhibitory function of MDSCs in A375 melanoma model, suggesting a potential strategy to augment the efficacy of combined immunotherapy.
\end{abstract}

Keywords: Preconditioning chemotherapy - immunomodulation - myeloid-derived suppressor cells - melanoma

Asian Pac J Cancer Prev, 16 (10), 4329-4333

\section{Introduction}

Myeloid-derived suppressor cells (MDSCs) are a heterogeneous population of cells deriving from the myeloid cell lineage and abnormally accumulating in the patients with various tumors (Crook et al., 2014). Gr-1 and CD11b, two primary cell surface markers, are usually used to identify the MDSCs (Medina-Echeverz et al., 2015). There is strong evidence that MDSCs could inhibit both adaptive and innate immunity mainly through their capacity to produce nitric oxide and radical oxygen species, thus leading to the inhibition of activation, proliferation and function of effector T cells (Fernandez et al., 2014). Given this immuno-inhibitory effect of MDSCs, it has been proposed that down-regulation of MDSCs may significantly augment the anti-tumor immune activity. Previous studies displayed that except for direct cytotoxic effects on tumor cells, some chemotherapeutic drugs could also eliminate MDSCs, resulting in enhanced anti-tumor response (Suzuki et al., 2005; Vincent et al., 2010; Chen et al., 2012; Alizadeh et al., 2014a; 2014b; Zheng et al., 2015). Based on the previous research results that cisplatin (DDP) could decrease the percentage of MDSCs in immuno-competent B16 melanoma model (Chen et al., 2012), the down-regulatory effect of DDP on MDSCs in immune-deficient nude mice bearing A375 human melanoma was further investigated in this study.

\section{Materials and Methods}

\section{General data}

Animals: SPF female BALB/c nude mice and BALB/c mice (6 8 weeks old) were purchased from Academy of Military Medical Science (Beijing, China) and housed in a pathogen-free animal facility with moderate temperature and humidity. The animal experiments were in accordance with the guide for care and use of laboratory animals as approved by Nanjing Medical University.

Cells lines: Human melanoma cell line A375 was obtained from Shanghai Institute of Biochemistry and Cell Biology (Shanghai, China) and cultured in complete RPMI 1640 medium (Hyclone, Waltham, MA, USA) supplemented with $10 \%$ fetal bovine serum. All cells were cultured in a humidified atmosphere with $5 \% \mathrm{CO}_{2}$ at $37^{\circ} \mathrm{C}$.

\section{Methods}

Tumor model: A375 cells $\left(10^{\wedge} 6 / 100 \mu \mathrm{L}\right.$ PBS $)$ were subcutaneously inoculated in the right flank of BALB/c nude mice. When tumors were detectable (about $5 \mathrm{~mm}$ in diameter), the mice were administrated with the single 
dose of $5 \mathrm{mg} / \mathrm{kg}$ DDP (Qilu Pharmaceutical Co., Ltd, China) as experimental group approximately 7 days after tumor challenge, whereas the mice received normal saline (NS) alone as control group. Besides, both experimental and control groups included 3 mice at least.

Flow cytometry: On the selected days after treatment, the single cell suspensions of peripheral blood and tumor tissue were prepared and stained with the indicated monoclonal antibodies. FITC-conjugated anti-CD11b, PE-conjugated anti-Gr-1, PE-Cy5-conjugated anti-CD3 and FITC-conjugated anti-CD8 antibodies were purchased from eBioscience (San Diego, CA, USA). To determine the percentage of MDSCs, the single cell suspension was subjected to flow cytometry and gated by plotting forward $v s$ side scatter followed by gating on $\mathrm{Gr}-1^{+} / \mathrm{CD} 11 \mathrm{~b}^{+}$cells. For the suspension from peripheral blood, 10,000 events were collected while 100,000 for the tumor tissue. All analyses were conducted using Accuri C6 software.

Co-culture system: $\mathrm{CD}^{+} / \mathrm{CD}^{+} \mathrm{T}$ cells were isolated from the spleen of naive BALB/c mice using MACS system. Then $\mathrm{CD} 11 \mathrm{~b}^{+}$MDSCs were also purified from the spleen of mice bearing A375 tumors using MACS system. These sorted $\mathrm{T}$ cells were mixed with enriched MDSCs that had been treated with either NS or single injection of $5 \mathrm{mg} / \mathrm{kg}$ DDP $48 \mathrm{~h}$ before isolation. The cell ratios were one MDSC for one T cell in triplicate. Soluble anti-CD3 (2 $\mu \mathrm{g} / \mathrm{mL}$ ), anti-CD28 (2 $\mu \mathrm{g} / \mathrm{mL})$ and interleukin-2 (IL-2, 1 $000 \mathrm{IU} / \mathrm{mL}$ ) obtained from PEPROTECH (Rocky Hill, NJ, USA) were added to the co-culture system. Stimulation was maintained for $24 \mathrm{~h}$.

Carboxyfluorescein succinimidyl ester (CFSE) labeling assay: The purified splenic $\mathrm{CD}^{+} / \mathrm{CD}^{+} \mathrm{T}$ cells were incubated with $5 \mu \mathrm{mol} / \mathrm{L}$ CFSE obtained from Dojindo Laboratories (Tokyo, Japan) for $10 \mathrm{~min}$ and then mixed with MDSCs in the co-culture system. After incubation for $24 \mathrm{~h}$, the cells were harvested and subjected to the flow cytometry for the determination of $\mathrm{CFSE}^{+}$cells.

Enzyme-linked immunospot (ELISPOT) assay: The secretion of interferon- $\gamma$ (IFN- $\gamma$ ) by T cells was detected by ELISPOT using Mouse ELISPOT kit (DAKEWEI, China) according to the manufacturer protocol. In brief, T cells $\left(1 \times 10^{5}\right.$ cells/well $)$ co-cultured with MDSCs were added into anti-IFN- $\gamma$ coated 96-well plates in triplicate, stimulated with anti-CD3 $(2 \mu \mathrm{g} / \mathrm{mL})$, anti-CD28 $(2 \mu \mathrm{g} /$ $\mathrm{mL})$ and IL-2 (1 $000 \mathrm{IU} / \mathrm{mL})$ for $24 \mathrm{~h}$. After washing steps, the secondary biotin-conjugated anti-IFN- $\gamma$ detection antibody and diluted streptavidin-HRP conjugate solution were added and incubated for $1 \mathrm{~h}$ successively. Finally, the plates were treated with AEC substrate solution and incubated at room temperature for $25 \mathrm{~min}$ in the dark. After developing, the spots were counted using an ELISPOT reader (Bioreader 4000; Bio-sys, Germany).

\section{Statistical data analysis}

Statistical analysis was conducted using SPSS 13.0 statistical software (Chicago, Illinois, USA). Measurement data were expressed as the mean \pm standard deviation $(\mathrm{x} \pm \mathrm{s})$ and compared with $\mathrm{t}$ test. Significant differences between groups were compared using ANOVA, and LSD was applied for multiple comparisons. Differences were considered to be significant at the time of $P<0.05$.

\section{Results}

$D D P$ reduced the frequency of $G r-1^{+} / C D 11 b^{+} M D S C s$ in the tumor tissue

In preliminary experiments, we found that DDP could eliminate $\mathrm{Gr}-1^{+} / \mathrm{CD} 11 \mathrm{~b}^{+}$MDSCs in B16 melanoma model (Chen et al., 2012). To investigate whether DDP had a similar effect on the frequency of MDSCs induced by other cancer cell lines, A375 human melanoma model was established and treated with the single dose of DDP $(5 \mathrm{mg} /$ $\mathrm{kg}$ ). In consistent with the data obtained in B16 melanoma model, the percentage of MDSCs elevated, which was associated with the increased tumor mass without DDP treatment (Figure 1). Administration of DDP was able to dramatically decrease these $\mathrm{Gr}-1^{+} / \mathrm{CD} 11 \mathrm{~b}^{+} \mathrm{MDSC}$ populations in mice bearing A375 tumor. Three days after DDP treatment, a typical flow cytometry trace showed a marked decrease in the percentage of MDSCs $(25.77 \pm 5.55$ vs $15.10 \pm 0.53, P=0.03)$. However, DDP-induced depletion seemed transient. The percentage of MDSCs rapidly rose to the level comparative to that in control group 6 and 9 d after DDP treatment.

DDP exerted no effect on the frequency of $\mathrm{Gr}-1^{+} / C D 11 b^{+}$ MDSCs in peripheral blood.

To determine whether circulating MDSCs were affected by DDP treatment, we also examined the percentage of MDSCs in peripheral blood in A375 melanoma model. As shown in Figure 2, the frequency of $\mathrm{Gr}-1^{+} / \mathrm{CD} 11 \mathrm{~b}^{+}$ MDSCs in peripheral blood of tumor-bearing hosts increased with the growth of tumor mass without DDP intervention. However, DDP treatment did not affect the frequency of circulating MDSCs in mice bearing A375

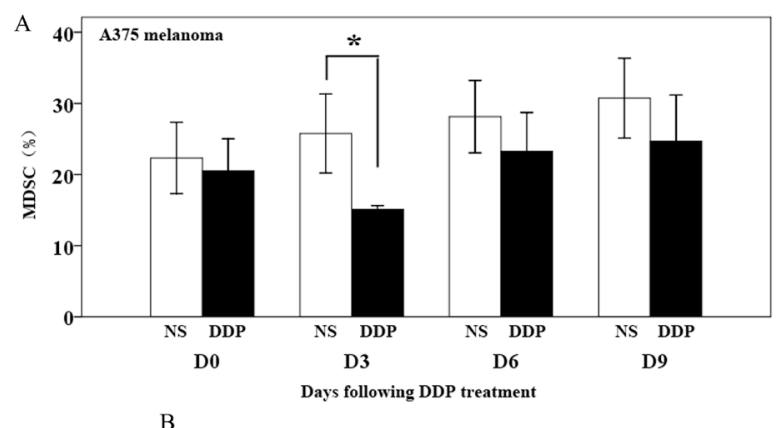

B

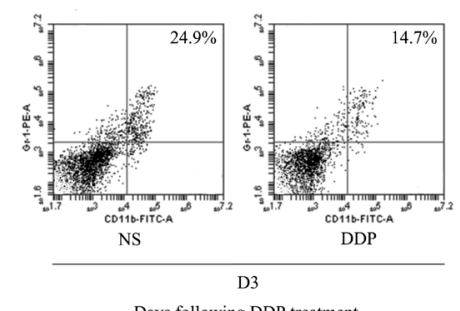

Days following DDP treatment

Figure 1. Depletion of $\mathrm{Gr}^{+} \mathbf{1}^{+} / \mathrm{CD} 11 \mathrm{~b}^{+}$MDSCs in the Tumor Tissue after DDP Treatment. Mice bearing A375 melanoma were treated with $5 \mathrm{mg} / \mathrm{kg}$ of DDP (i.p., D0) or left untreated. Tumor tissues were isolated and prepared for the single cell suspension on D0, 3, 6, 9 after DDP treatment. The percentage of $\mathrm{Gr}-1^{+} / \mathrm{CD} 11 \mathrm{~b}^{+}$MDSCs in the tumor tissue were determined through cytometry (A) and the typical data that the experiment was shown (B). Columns: the mean of triplicate measurements; Bars: SE; $* P<0.05$ 


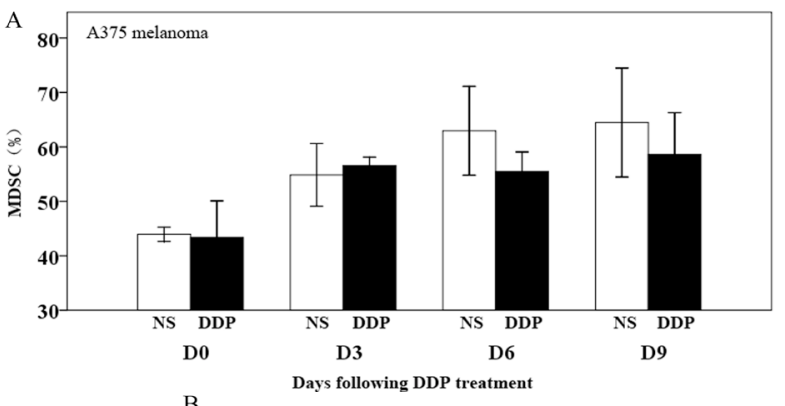

B

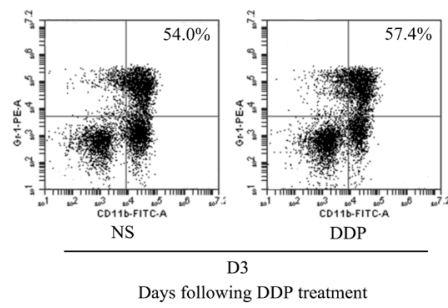

Figure 2. Frequency of Gr-1 ${ }^{+} / \mathrm{CD}^{11 b^{+}}$MDSCs in Peripheral Blood after DDP Treatment. Mice bearing A375 melanoma were treated with $5 \mathrm{mg} / \mathrm{kg}$ of DDP (i.p., D0) or left untreated. Peripheral blood was isolated and prepared for the single cell suspension on D0,3,6,9 after treatment. The percentage of $\mathrm{Gr}-1^{+} / \mathrm{CD} 11 \mathrm{~b}^{+}$MDSCs in the peripheral blood was determined through cytometry (A) and the typical data that the experiment was shown (B). Columns: the mean of triplicate measurements; Bars: SE

melanoma. Although there was a mild decline regarding the percentage of MDSCs compared with control group, significant difference was not presented.

DDP enhanced the proliferation of $T$ cells co-cultured with MDSCs

Previous studies have reported that MDSCs could suppress the anti-tumor activity of $\mathrm{CD}^{+} \mathrm{T}$ cells (Umansky et al., 2013; Ostrand-Rosenberg et al., 2010). We speculated that except for the down-regulatory effect on the frequency of MDSCs, DDP treatment could also attenuate the immuno-inhibitory effect of MDSCs on T cells. $\mathrm{CD}^{+} / \mathrm{CD}^{+}{ }^{+}$cells were purified from $\mathrm{BALB} / \mathrm{c}$ mice and stained with CFSE. To determine the suppressive effect of MDSCs on the proliferation of T cells, we mixed the sorted $\mathrm{CD}^{+} / \mathrm{CD}^{+} \mathrm{T}$ cells with $\mathrm{CD} 11 \mathrm{~b}^{+}$MDSCs from the mice bearing A375 melanoma that had been treated $3 \mathrm{~d}$ previously with DDP or NS. As shown in Figure 3, untreated MDSCs could significantly reduce the proliferation of $\mathrm{T}$ cells $(4.06 \pm 0.94$ vs $9.87 \pm 1.60$, $P=0.001$ ), whereas DDP treatment partially abrogated this immuno-inhibitory effect of MDSCs. The proliferation of co-cultured T cells profoundly elevated $(6.52 \pm 0.79$ vs $4.96 \pm 0.94, P=0.025)$, but was still markedly inhibited by comparison to that without MDSCs $(6.52 \pm 0.0 .79 \mathrm{vs}$ $9.87 \pm 1.60, P=0.031)$.

DDP augmented the secretion of IFN- $\gamma$ by $T$ cells cocultured with MDSCs.

Whether DDP treatment could alter the inhibitory effect of MDSCs on IFN- $\gamma$ production of T cells was also investigated. After co-culture with untreated MDSCs in A375 melanoma-bearing mice, the secretion of IFN- $\gamma$ by

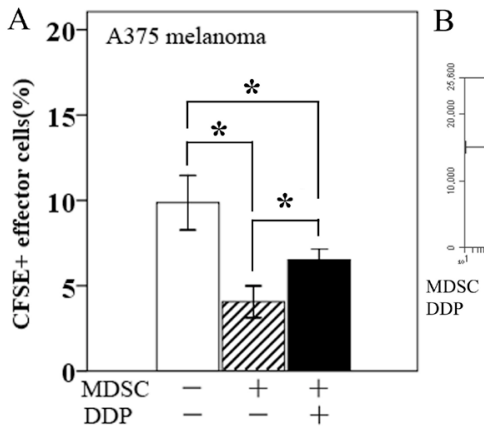

B
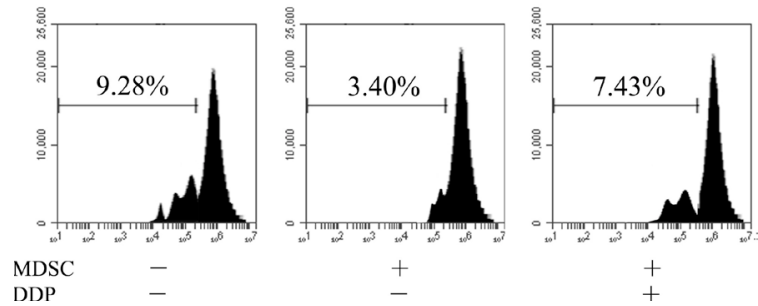

Figure 3. Enhanced Proliferation of T Cells CoCultured with MDSCs after DDP Treatment. A375 melanoma-bearing mice were administered with DDP $5 \mathrm{mg} / \mathrm{kg}$ (i.p., D0) or left untreated. $3 \mathrm{~d}$ after DDP treatment, the spleen tissues were isolated from the mice and CD11b $\mathrm{b}^{+}$MSCs were sorted by flow cytometry. The obtained MDSCs were then cocultured with $\mathrm{CFSE}^{+} \mathrm{T}$ cells for $24 \mathrm{~h}$. The proliferation of T cells was subjected to flow cytometry (A) and the typical data that the experiment was shown (B). Columns: the mean of triplicate measurements; Bars: SE; $* \mathrm{P}<0.05$

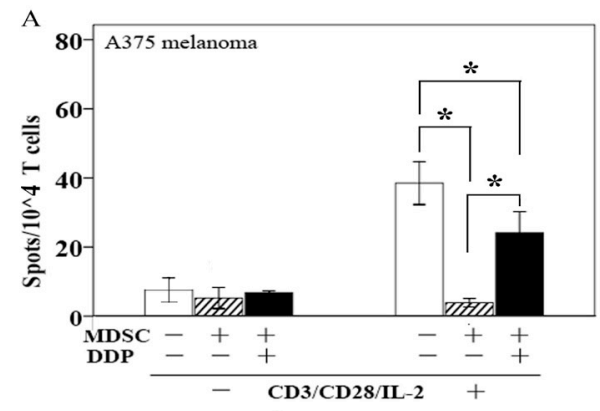

B

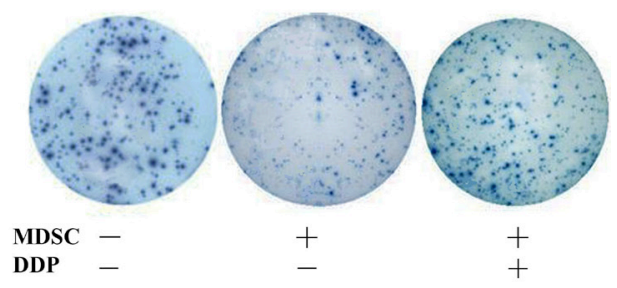

Figure 4. Augmented Secretion of IFN- $\gamma$ by T Cells Co-Cultured with MDSCs after DDP Treatment. A375 melanoma-bearing mice were administered with DDP $5 \mathrm{mg} / \mathrm{kg}$ (i.p., D0) or left untreated. $3 \mathrm{~d}$ after DDP treatment, the spleen tissues were isolated from the mice and CD11 $\mathrm{b}^{+}$MDSCs were sorted by flow cytometry. The obtained MDSCs were then cocultured with T cells for $24 \mathrm{~h}$. The IFN- $\gamma$ production of T cells was detected by ELISPOT (A) and the typical data that the experiment was shown $(\mathbf{B})$. Columns: the mean of triplicate measurements; Bars: SE; *: P<0.05

T cells was obviously inhibited ( $3.80 \pm 1.22$ vs $38.40 \pm 6.20$, $P=0.0007)$, whereas DDP treatment partially reversed this immuno-suppressive effect of MDSCs, resulting in 
significantly increased IFN- $\gamma$ production by co-cultured T cells $(24.06 \pm 6.10$ vs $3.80 \pm 1.22, P=0.005)$. However, the secretion of IFN- $\gamma$ by T cells remained in a decreased status compared with that without MDSCs $(24.06 \pm 6.10$ vs $38.40 \pm 6.20, P=0.046$ ).

\section{Discussion}

In recent years, immunotherapy has displayed a great promise in the treatment of various tumors (Su et al., 2013; Zhang et al., 2014a; Wang et al., 2014b; Anagnostou et al., 2015; Curti et al., 2015; Swaika et al., 2015). However, the therapeutic activity of immunotherapy is limited by the immunosuppressive factors active in the tumor hosts. It is increasingly recognized that manipulation of the host immune system is essential to guarantee the anti-tumor effect of immunotherapy (Shekarian et al., 2015). As a population of immature myeloid cells, MDSCs possess the ability to inhibit $\mathrm{T}$ cell antigen-specific response mainly through the production of nitric oxide and radical oxygen species, consequently leading to the failure of anti-tumor activity (Fernandez et al., 2014). It has been proposed that elimination of MDSCs will be an effective approach to abrogate the tumor-induced immune-suppression and enhance the activity of immunotherapy. Accordingly, a number of strategies have been evaluated. Among which, chemotherapeutic drugs are demonstrated to have the ability to selectively eliminate MDSCs.

It was reported that both gemcitabine and 5-fluorouracil could reduce the number of splenic $\mathrm{Gr}-1^{+} / \mathrm{CD} 11 \mathrm{~b}^{+}$MDSCs while preserving the number of T cells, NK cells and $\mathrm{B}$ cells, accompanied by an increased anti-tumor activity of $\mathrm{CD}^{+} \mathrm{T}$ cells and NK cells (Suzuki et al., 2005; Vincent et al., 2010; Chen et al., 2012; Alizadeh et al., 2014a; Alizadeh et al., 2014b; Zheng et al., 2015). Besides, DDP could deplete the splenic Gr-1+CD $11 b^{+}$MDSCs in murine B16 melanoma (Chen et al., 2012). To date, there are few studies that delineate the function of MDSCs after treatment of chemotherapeutic drugs, even fewer studies on the change of MDSCs induced by chemotherapeutic drugs in immune-deficient mice bearing human tumors. Therefore, in this study, BALB/c nude mice bearing human melanoma were established, and the frequency and function of MDSCs after DDP treatment were also analyzed. The research results displayed that DDP $(5 \mathrm{mg} /$ $\mathrm{kg}$ ) could significantly reduce the frequency of MDSCs in the tumor tissue in mice bearing A375 human melanoma. However, this depletion was transient and only lasted for $3 \mathrm{~d}$ after DDP treatment, indicating that there may be an optimal time point when the combined regimen exerts the maximal synergistic anti-tumor activity. Moreover, DDPinduced depletion of MDSCs frequency was not detected in peripheral blood. We speculated that the elimination of MDSCs induced by DDP was tumor cells-dependent. Some cytokines released by tumor cells, such as IL-6, IL-10 and VEGF, could recruit MDSCs to migrate and accumulate into the tumor tissue (Gabrilovich et al., 2009). DDP was presumed to inhibit the release of these soluble factors by tumor cells, thus inducing the decline of MDSCs in tumor tissue rather than in peripheral blood.

In addition to showing a decreased frequency of
MDSCs in the tumor tissue of A375 melanoma-bearing mice after DDP treatment, it was also found a concomitant decrease in the immuno-inhibitory activity of MDSCs. DDP treatment attenuated the immunosuppressive effect of MDSCs on the proliferation and IFN- $\gamma$ production of T cells. Notably, DDP treatment only partially reversed the immuno-inhibitory effect of MDSCs, the proliferation and IFN- $\gamma$ production by $\mathrm{T}$ cells still went down profoundly by comparison to that without MDSCs. The mechanisms on whether DDP can hinder the immuno-inhibitory effect of MDSCs remain elusive. One speculation may be that MDSCs can differentiate into dendritic cells after DDP treatment. Cyclophosphamide is reported to induce a marked expansion of immature dendritic cells which share the same progenitor with MDSCs (Salem et al., 2009). However, further studies are still needed to elucidate the underlying mechanisms.

In this study, the down-regulatory effect of DDP on MDSCs was observed in nude mice bearing human A375 melanoma. Nevertheless, the murine immune environment was not able to mimic the human immune background. Although chemotherapeutic drugs were capable of decreasing MDSCs in mice, an interesting question that remains to be solved is that whether the immune-modulating effect of chemotherapeutic drugs could be translated into clinical practice.

In summary, DDP can decrease the frequency and attenuate immuno-inhibitory function of MDSCs in A375 melanoma model, suggesting a potential strategy to augment the efficacy of combined immunotherapy.

\section{Acknowledgements}

This study was supported by a grant from National Natural Science Foundation of China (NSFC 81201597).

\section{References}

Alizadeh D, Larmonier N (2014a). Chemotherapeutic targeting of cancer-induced immunosuppressive cells. Cancer Res, 74, 2663-8.

Alizadeh D, Katsanis E, Larmonier N (2014b). Chemotherapeutic targeting of myeloid-derived suppressor cells. Oncoimmunol, 3, 27359.

Anagnostou VK, Brahmer JR (2015). Cancer immunotherapy: a future paradigm shift in the treatment of non-small cell lung cancer. Clin Cancer Res, 21, 976-84.

Chen J, Huang X, Huang G, et al (2012). Preconditioning chemotherapy with cisplatin enhances the antitumor activity of cytokine-induced killer cells in a murine melanoma model. Cancer Biother Radiopharm, 27, 210-20.

Crook KR, Liu P (2014). Role of myeloid-derived suppressor cells in autoimmune disease. World J Immunol, 4, 26-33.

Curti BD, Urba WJ (2015). Clinical deployment of antibodies for treatment of melanoma. Mol Immunol, [Epub ahead of print].

Fernández A, Oliver L, Alvarez R, et al (2014). Adjuvants and myeloid-derived suppressor cells: enemies or allies in therapeutic cancer vaccination. Hum Vaccin Immunother, 10, 3251-60.

Gabrilovich DI, Nagaraj S (2009). Myeloid-derived suppressor cells as regulators of the immune system. Nat Rev Immunol, 9, 162-74.

Medina-Echeverz J, Ma C, Duffy A, et al (2015). Systemic 

agonistic anti-CD40 treatment of tumor bearing mice modulates hepatic myeloid suppressive cells and causes immune-mediated liver damage. Cancer Immunol Res, [Epub ahead of print].

Ostrand-Rosenberg S (2010). Myeloid-derived suppressor cells: more mechanisms for inhibiting antitumor immunity. Cancer Immunol Immunother, 59, 1593-600.

Salem ML, Diaz-Montero CM, Al-Khami AA, et al (2009). Recovery from cyclophosphamide-induced lymphopenia results in expansion of immature dendritic cells which can mediate enhanced prime-boost vaccination antitumor responses in vivo when stimulated with the TLR3 agonist poly (I:C). J Immunol, 182, 2030-40.

Shekarian T, Valsesia-Wittmann S, Caux C, et al (2015). Paradigm shift in oncology: targeting the immune system rather than cancer cells. Mutagenesis, 30, 205-11.

Suzuki E, Kapoor V, Jassar AS, et al (2005). Gemcitabine selectively eliminates splenic $\mathrm{Gr}-1^{+} / \mathrm{CD} 11 \mathrm{~b}^{+}$myeloid suppressor cells in tumor-bearing animals and enhances antitumor immune activity. Clin Cancer Res, 11, 6713-21.

Swaika A, Hammond WA, Joseph RW (2015). Current state of anti-PD-L1 and anti-PD-1 agents in cancer therapy. Mol Immunol, [Epub ahead of print].

Su S, Zhou H, Xue M, et al (2013). Anti-tumor efficacy of a hepatocellular carcinoma vaccine based on dendritic cells combined with tumor-derived autophagosomes in murine models. Asian Pac J Cancer Prev, 14, 3109-16.

Umansky V, Sevko A (2013). Tumor microenvironment and myeloid-derived suppressor cells. Cancer Microenviron, 6, 169-77.

Vincent J, Mignot G, Chalmin F, et al (2010). 5-Fluorouracil selectively kills tumor-associated myeloid-derived suppressor cells resulting in enhanced $\mathrm{T}$ cell-dependent antitumor immunity. Cancer Res, 70, 3052-61.

Wang WJ, Qin SH, Zhang JW, et al (2014). Combination doxorubicin and interferon- $\alpha$ therapy stimulates immunogenicity of murine pancreatic cancer Panc02 cells via up-regulation of NKG2D ligands and MHC class I. Asian Pac J Cancer Prev, 15, 9667-72.

Zhang QM, Shen N, Xie S, et al (2014). MAGED4 expression in glioma and upregulation in glioma cell lines with 5-aza2'-deoxycytidine treatment. Asian Pac J Cancer Prev, 15, 3495-501.

Zheng Y, Dou Y, Duan L, et al (2015). Using chemo-drugs or irradiation to break immune tolerance and facilitate immunotherapy in solid cancer. Cell Immunol, 294, 54-9. 